\title{
Early and long-term outcomes of bioresorbable vascular scaffolds in the treatment of patients with coronary artery disease in real-world clinical practice - insights from the ZABRZE-BVS registry
}

\author{
Jacek Piegza ${ }^{1}$, Piotr Desperak ${ }^{1}$, Jacek Kowalczyk², Marek Gierlotka ${ }^{1}$, Michat Hawranek ${ }^{1}$, Piotr Chodór², \\ Marcin Świerad ${ }^{2}$, Andrzej Lekston ${ }^{1}$, Zbigniew Kalarus², Mariusz Gąsior ${ }^{1}$ \\ ${ }^{1} 3^{\text {rd }}$ Chair and Department of Cardiology, Medical University of Silesia in Katowice, School of Medicine with the Division of Dentistry \\ in Zabrze, Silesian Center for Heart Diseases, Poland \\ 2Department of Cardiology, Congenital Heart Diseases and Electrotherapy, Medical University of Silesia in Katowice, School of Medicine \\ with the Division of Dentistry in Zabrze, Silesian Center for Heart Diseases, Poland
}

Adv Interv Cardiol 2018; 14, 4 (54): 338-346

DOI: https://doi.org/10.5114/aic.2018.79864

\begin{abstract}
Introduction: Randomized trials have proven the feasibility and safety of the bioresorbable vascular scaffold (BVS) in selected populations of patients. Data concerning the results of BVS in "real-world" registries with an appropriate sample size are limited.

Aim: Assessment of early- and long-term outcomes of patients undergoing bioresorbable scaffold implantation in an all-comers population of the ZABRZE-BVS registry.

Material and methods: The ZABRZE-BVS registry is a prospective registry including consecutive patients treated in the period 2013-2016 with the intention to implant a BVS (ABSORB, Abbott Vascular, Santa Clara, California). The primary endpoint was occurrence of the 12- and 24-month device-oriented composite endpoint (DoCE) defined as cardiac death, target-vessel myocardial infarction (TV-MI) or target lesion revascularization (TLR). The secondary endpoint includes occurrence of patient-oriented composite endpoint (PoCE) at 12 and 24 months, device (lesion basis) and procedural success (patient basis).

Results: A total of 456 patients during 467 procedures received 588 scaffolds in 563 lesions. Of note, $25.4 \%$ of patients presented with diabetes mellitus and $62.3 \%$ had an acute coronary syndrome. In QCA analysis, $78.7 \%$ of patients had type B2/C lesions, minimal lumen diameter was $0.78 \pm 0.54 \mathrm{~mm}$, whereas post-procedural acute lumen gain was $1.61 \pm 0.61 \mathrm{~mm}$. Median follow-up was 781 days. The cumulative rate of DoCE was $6.7 \%$ at 12 months and $12.2 \%$ at 24 months. Rates of 12 - and 24-month PoCE were $12.4 \%$ and $20.1 \%$, respectively. The percentage of device success was $98.7 \%$, while the procedural success rate was $96.9 \%$.

Conclusions: The Absorb BVS was successfully and safely implanted in an unselected group of patients. Scaffold thrombosis developed predominantly in patients with acute coronary syndrome (ACS).
\end{abstract}

Key words: bioresorbable vascular scaffold, coronary percutaneous intervention, long-term survival.

S u m m a ry

Randomized studies comparing bioresorbable vascular scaffold (BVS) to drug-eluting stents (DES) have shown a greater risk of complications in patients with implanted scaffolds, calling the advantages of using the Absorb BVS into question. Based on these reports of an increased risk of scaffold thrombosis, routine implantation of the Absorb BVS was abandoned, followed by the company ceasing sale of the product. This makes the surveillance of around 150,000 patients with the Absorb implanted important. Early and long-term clinical outcomes are reported for this all-comers single-center registry of the Absorb BVS. This registry shows the "real-world" clinical practice of BVS implantation in our institution. Our primary endpoint device-oriented composite endpoint (DoCE) was $6.7 \%$ at 12 months and $12.2 \%$ and 24 months. The Absorb BVS was successfully and safely implanted in an unselected group of patients. Scaffold thrombosis developed predominantly in patients with acute coronary syndrome (ACS). Despite the complexity of the lesion and high number of patients with ACS, the occurrence of scaffold thrombosis was acceptable, especially in comparison to other published registries and randomized trials.

\section{Corresponding author:}

Piotr Desperak MD, $3^{\text {rd }}$ Chair and Department of Cardiology, Medical University of Silesia in Katowice, School of Medicine with the Division of Dentistry in Zabrze, Silesian Center for Heart Diseases, 2 Szpitalna St, 41-800 Zabrze, Poland, phone: +48 514 374 167,

e-mail: piotr.desperak@op.pl

Received: 4.05.2018, accepted: 14.08.2018. 


\section{Introduction}

The current generation of drug-eluting stents (DES) has demonstrated a higher level of safety and efficacy in treating coronary lesions than preceding generations of DES and bare metal stents (BMS) [1, 2]. This effect was achieved by improving biocompatibility using cobalt-chromium construction, thinner stent struts and biodegradable polymers [3, 4]. Despite this, drug-eluting stents still have some limitations. Implantation of a permanent coronary metallic prosthesis can lead to stent thrombosis, restenosis and malapposition. The new bioresorbable technologies were supposed to solve the problem. The fundamental basis for developing them was to reduce neointimal hyperplasia, neo-atherosclerosis and late thrombosis. Initial studies have demonstrated the safety and efficacy of the Absorb BVS (Abbott Vascular, Santa Clara, California) in relatively simple lesions [5-7]. Subsequent randomized studies comparing bioresorbable vascular scaffolds (BVS) to DES have shown a greater risk of complications in patients with implanted scaffolds, calling the advantages of using the Absorb BVS into question [8]. Based on these reports of an increased risk of scaffold thrombosis, routine implantation of the Absorb BVS was abandoned, followed by the company ceasing sale of the product. This makes the surveillance of around 150,000 patients with the Absorb implanted important. The Abbott company advises the follow-up of patients in existing clinical studies as per protocols.

\section{Aim}

In accordance with good clinical practice, the Silesian Centre for Heart Diseases in Zabrze (a high-volume center performing yearly nearly 2500 percutaneous coronary interventions $(\mathrm{PCI})$ ) set up a detailed registry from the initiation of Absorb BVS implantation. The aim of this registry was to evaluate both early and long-term efficacy and safety outcomes in patients subjected to percutaneous coronary interventions with the Absorb BVS. In addition, the influence of the learning curve of BVS implantation on in-hospital and long-term outcomes was analyzed.

\section{Material and methods}

The ZABRZE-BVS registry is prospective, all-comers, single-center registry including consecutive patients treated with percutaneous coronary intervention between June 2013 and December 2016 with the intention to implant a BVS (ABSORB, Abbott Vascular, Santa Clara, California). Implantation procedures were performed in a highly specialized cardiology center with both on-site 24 h/day catheterization duty and cardiac surgery.

The management of the study population was in accordance with the recommendations of the European Society of Cardiology (ESC) [9]. Before the procedure, loading doses of acetylsalicylic acid, and weight-adjusted unfractionated heparin were administered. A P2Y12 inhibitor was given before coronary angiography in all acute coronary syndrome (ACS) patients and in all patients with stable angina (SA) undergoing angioplasty. In all cases, coronary angiography with standard techniques and equipment was performed. The decision of access site (radial, femoral or other) and the type of diagnostic catheters was taken by the operator. Evaluation of the coronary arteries was made based on visual assessment and online quantitative coronary angiography (QCA). Where appropriate, the use of intravascular imaging (intravascular ultrasound or optical coherence tomography) was encouraged. All therapeutic decisions including balloon pre-dilatation and post-dilatation, use of stents, type of stents, glycoprotein Ilb/IIla receptor inhibitors and other established interventional techniques were at the operator's discretion.

Management decisions were based on contemporary knowledge of the data on the BVS implantation technique including: 1) an accurate measurement of the diameter of the treated segment using QCA at maximum extension of the treated vessel (via intracoronary administration of nitroglycerin); 2) optimal preparation of the lesion with manual thrombectomy and selection of appropriate type and size of the balloon for pre-dilatation to obtain residual stenosis less than $40 \%$ of diameter stenosis; 3) BVS implantation technique with a gradual increase ( $2 \mathrm{~atm}$ per $5 \mathrm{~s}$ ) and maintenance of target pressure of the expanded balloon for $30 \mathrm{~s}$; 4) post-dilatation with a balloon diameter of not more than $0.5 \mathrm{~mm}$ from the nominal diameter of the stent.

After the procedure, patients with ACS were transferred to the intensive cardiac care unit. In case of recurrence of ischemia after index $\mathrm{PCl}$, urgent coronary angiography was performed and appropriate treatment provided. In patients with multi-vessel disease, additional revascularization procedures were performed during index hospitalization or routinely planned up to a maximum of 3 months after discharge. After discharge, dual antiplatelet therapy was recommended for at least 12 months. Furthermore, each patient had prescribed a standard secondary prevention in accordance with ESC guidelines [9].

Additionally, quantitative coronary angiography (QCA) was performed by an independent Core Lab (Krakow Cardiovascular Research Institute, KCRI, Krakow, Poland) for the first 224 patients who had a BVS implanted.

Baseline clinical and angiographic data of all patients enrolled in the ZABRZE-BVS Registry were recorded in the institutional database. Information on long-term outcomes, including all-cause deaths with exact dates, reasons for re-hospitalization, and procedures were obtained from the official records of the National Health Fund, the single public compulsory health insurer in Poland. Death 
was considered as cardiac unless a non-cardiac reason was confirmed. Therefore, follow-up data were available for all patients enrolled. The registry was granted ethics approval by the Institutional Review Board and the Bioethics Committee of the University, and is consistent with the ethical standards laid down in the 1964 Declaration of Helsinki and its amendments.

Clinical endpoints used in the ZABRZE-BVS Registry were consistent with Academic Research Consortium (ARC) consensus Clinical End Points in Coronary Stent Trials [10]. Briefly, the primary endpoint encompassed device-oriented cardiovascular endpoint (DoCE) defined as cardiac death, target-vessel myocardial infarction (TV-MI) and ischemia-driven target lesion revascularization (ID-TLR). Secondary endpoints included device success (per lesion), procedural success (per patient), scaffold/stent thrombosis (ST) and patient-oriented cardiovascular endpoint (PoCE) defined as all-cause death, all non-fatal myocardial infarction, and all ischemia-driven revascularization. Detailed definitions of the above clinical endpoints and ST have been previously described in the ARC statement [10]. Device success was defined as successful delivery and deployment of the scaffold/stent at the intended target lesion and successful withdrawal of the delivery system with attainment of final in-scaffold/stent residual stenosis of $<20 \%$. Procedure success was defined as achievement of device success in all intended-to-treat lesions without the occurrence of cardiac death, TV-MI, or repeat ID-TLR during the hospital stay. In the multiple target lesion setting, all lesions must meet clinical procedure success criteria to have a patient-level procedure success.

\section{Statistical analysis}

A comparison of baseline and angiographic characteristics, in-hospital, early and long-term outcomes depending on clinical manifestation of coronary artery disease (CAD) was performed. Additionally, a comparison between patients treated in the first period (between June 2013 and March 2015) and the second period (between April 2015 and December 2016) was made. Normality of distribution was verified by the Shapiro-Wilk test. Continuous variables were summarized using the arithmetic mean with standard deviation (SD) or median with quartiles 1 and $3(\mathrm{Q} 1-\mathrm{Q} 3)$. The analysis of Student's t-test for comparison of continuous parameters with normal distribution was performed, whereas the Mann-Whitney $U$-test for parameters with non-normal distribution was used. Categorical variables were compared using the $\chi^{2}$ Pearson's test with the Yates correction if the expected number of observations was less than 5 . Primary, secondary and clinical endpoints were analyzed using the Kaplan-Meier method with the log-rank test. A two-sided $p$-value $<0.05$ was considered significant. The Statistica
12 software (StatSoft Inc., Tulsa, Oklahoma) was used for all calculations.

\section{Results}

A total of 456 patients were enrolled in the study. Table I summarizes the baseline demographics of the overall study population. The mean age of the patients was $57.3 \pm 9.8$ years and $70.8 \%$ were men (Table I). Of note, $25.4 \%$ of patients presented with diabetes mellitus and $68.9 \%$ had arterial hypertension. $27.9 \%$ of patients had a history of previous $\mathrm{MI}$ and $36.6 \%$ had previous revascularization ( $\mathrm{PCl}$ or coronary artery body graft (CABG)). The main indications for angiography were acute coronary syndromes (62.3\%) (ST segment (STEMI), non-ST segment elevation myocardial infarction or unstable angina (NSTE-ACS)).

During 467 procedures 588 scaffolds were implanted in 563 lesions. On average, the number of Absorb BVS implanted per patient was $1.29 \pm 0.56$. Single-vessel coronary artery disease was diagnosed in $63.8 \%$ of patients (Table I). Intervention in more than one lesion was performed in $19.3 \%$ of patients. The most frequently treated artery was the left anterior descending (LAD) (53.6\%). Aorto-ostial lesions occurred in $7.3 \%$ of cases and bifurcation lesions were found in $16.2 \%$ of patients (Table II). The percentage of device success was $98.7 \%$, while the procedural success rate was $96.9 \%$.

We performed a comparative analysis of two periods of BVS implantation: the first with 224 (49.1\%) patients and second with 232 (50.9\%) patients. QCA data were available in 224 patients, treated in the first period ( $\mathrm{Ta}$ ble III). As shown in Table IV, there were some significant differences between the second and the first period (QCA group). There were more patients with single vessel disease $(68.3 \%$ vs. $59.5 \%, p=0.05)$ and fewer patients with prior $\mathrm{PCl}(26.8 \%$ vs. $36.6 \%, p=0.024)$ in the first period than in the second. As presented in Table II, the number of lesions per patient as well as the number of scaffolds per patient was lower in the first period than in the second (respectively, lesions per patient: $1.13 \pm 0.49$ vs. 1.34 \pm 0.54 and number of scaffolds per patient: $1.14 \pm 0.49$ vs. $1.43 \pm 0.61$, both with $p<0.0001$ ). Primary, secondary and clinical endpoints did not differ between the first and second periods.

Median follow-up was 781 (quartile 1 to 3: 508 to 1029) days. The primary endpoint was occurrence of 12and 24-month DoCE. The cumulative rate of DoCE was $6.7 \%$ at 12 months and $12.2 \%$ at 24 months (Table V). At 12 months, the rate of cardiac death was $2.1 \%$, target vessel MI 4.4\% and TLR 4.7\%. The corresponding biennial rates for cardiac death, TV-MI and target vessel revascularization were $4.7 \%, 5.5 \%$ and $7.9 \%$. The secondary endpoint included occurrence of patient-oriented composite endpoint (PoCE) at 12 and 24 months. Rates of 12- and 24-month PoCE were $12.2 \%$ and $20.1 \%$ respectively (Ta- 
Table I. Baseline characteristics of the study population

\begin{tabular}{|c|c|c|c|c|}
\hline Factor & $\begin{array}{l}\text { Study population } \\
\qquad(N=456)\end{array}$ & $\begin{array}{l}\text { QCA group } \\
(N=224)\end{array}$ & $\begin{array}{l}\text { Non-QCA group } \\
\quad(N=232)\end{array}$ & $P$-value \\
\hline Age, mean \pm SD $(n / N)$ [years] & $57.3 \pm 9.8(456 / 456)$ & $57.7 \pm 10.2(224 / 224)$ & $57.0 \pm 9.4(232 / 232)$ & 0.44 \\
\hline Male, \% $(n / N)$ & $70.8(323 / 456)$ & $67.4(151 / 224)$ & $74.1(172 / 232)$ & 0.11 \\
\hline STEMI, \% $(n / N)$ & $27.0(123 / 456)$ & $26.8(60 / 224)$ & $27.2(63 / 232)$ & 0.93 \\
\hline NSTEMI, \% $(n / N)$ & $19.9(91 / 456)$ & $20.5(46 / 224)$ & $19.4(45 / 232)$ & 0.76 \\
\hline UA, \% $(n / N)$ & $15.4(70 / 456)$ & $13.8(31 / 224)$ & $16.8(39 / 232)$ & 0.38 \\
\hline $\mathrm{SA}, \%(n / N)$ & $37.7(172 / 456)$ & $38.8(87 / 224)$ & $36.6(85 / 232)$ & 0.63 \\
\hline Arterial hypertension, \% $(n / N)$ & $68.9(314 / 456)$ & $69.6(156 / 224)$ & $68.1(158 / 232)$ & 0.72 \\
\hline Prior MI, \% $(n / N)$ & $27.9(127 / 456)$ & $24.6(55 / 224)$ & $31.0(72 / 232)$ & 0.12 \\
\hline Prior PCI, \% $(n / N)$ & $31.8(145 / 456)$ & $26.8(60 / 224)$ & $36.6(85 / 232)$ & 0.024 \\
\hline Prior CABG, \% $(n / N)$ & $4.8(22 / 456)$ & $5.4(12 / 224)$ & $4.3(10 / 232)$ & 0.60 \\
\hline Atrial fibrillation, \% $(n / N)$ & $9.4(43 / 456)$ & $10.7(24 / 224)$ & $8.2(19 / 232)$ & 0.36 \\
\hline Peripheral artery disease, $\%(n / N)$ & $16.9(77 / 456)$ & $17.4(39 / 224)$ & $16.4(38 / 232)$ & 0.77 \\
\hline Diabetes mellitus, \% ( $n / N)$ & $25.4(116 / 456)$ & $23.7(53 / 224)$ & $27.2(63 / 232)$ & 0.39 \\
\hline Dyslipidemia, \% (n/N) & $70.0(319 / 456)$ & $67.0(150 / 224)$ & $72.8(169 / 232)$ & 0.17 \\
\hline Chronic kidney disease, \% $(n / N)$ & $8.3(38 / 456)$ & $9.4(21 / 224)$ & $7.3(17 / 232)$ & 0.43 \\
\hline Obesity, \% $(n / N)$ & $12.1(55 / 456)$ & $8.9(20 / 224)$ & $15.1(35 / 232)$ & 0.044 \\
\hline Current cigarette smoking, \% $(n / N)$ & $27.2(124 / 456)$ & $22.3(50 / 224)$ & $31.9(74 / 232)$ & 0.022 \\
\hline Cardiac arrest on admission, $\%(n / N)$ & $2.4(11 / 456)$ & $2.7(6 / 224)$ & $2.2(5 / 232)$ & 0.72 \\
\hline Killip class IVa $\%$ (n/N) & $1.7(8 / 456)$ & $1.3(3 / 224)$ & $2.2(5 / 232)$ & 0.51 \\
\hline WBCa , median, Q1-Q3 $(n / N)\left[\times 10^{3} / \mu \mathrm{l}\right]$ & $8.4,6.6-10.5(448 / 456)$ & $8.4,6.6-10.3(221 / 224)$ & $8.5,6.7-10.7(227 / 232)$ & 0.65 \\
\hline Hemoglobin ${ }^{\mathrm{a}}$, mean $\pm \mathrm{SD}(n / \mathrm{N})[\mathrm{mmol} / \mathrm{l}]$ & $8.9 \pm 1.0(448 / 456)$ & $8.9 \pm 1.0(221 / 224)$ & $9.0 \pm 0.9(227 / 232)$ & 0.40 \\
\hline Serum creatinine ${ }^{a}$, median, Q1-Q3 $(n / N)[\mu \mathrm{mol} / /]$ & $78,67-93(443 / 456)$ & $76,66-91(220 / 224)$ & $78,68-94(223 / 232)$ & 0.19 \\
\hline eGFRa , median, Q1-Q3 $(n / \mathrm{N})\left[\mathrm{ml} / \mathrm{min} / 1.73 \mathrm{~m}^{2}\right]$ & $87,74-104(441 / 456)$ & $88,75-105(216 / 172)$ & $85,73-102(225 / 456)$ & 0.53 \\
\hline eGFR $<60 \mathrm{ml} / \mathrm{min} / 1.73 \mathrm{~m}^{2 \mathrm{a}}, \%(n / \mathrm{N})$ & $11.3(50 / 441)$ & $10.6(23 / 216)$ & $12.0(27 / 225)$ & 0.65 \\
\hline LVEFa $^{2} \%$, mean \pm SD $(n / N):$ & $47.4 \pm 9.3(456 / 456)$ & $48.1 \pm 9.3(224 / 224)$ & $46.7 \pm 9.4(232 / 232)$ & 0.091 \\
\hline LVEF > 50\%a, \% $(n / N)$ & $52.4(239 / 456)$ & $56.7(127 / 224)$ & $48.3(112 / 232)$ & 0.072 \\
\hline LVEF 50-35\%a, \% (n/N) & $36.2(165 / 456)$ & $32.6(73 / 224)$ & $39.7(92 / 232)$ & 0.12 \\
\hline LVEF $<35 \%{ }^{\mathrm{a}}, \%(n / N)$ & $11.4(52 / 456)$ & $10.7(24 / 224)$ & $12.1(28 / 232)$ & 0.65 \\
\hline \multicolumn{5}{|l|}{ Coronary angiography, \% $(n / N)$ : } \\
\hline Single-vessel CAD & $63.8(291 / 456)$ & $68.3(153 / 224)$ & $59.5(138 / 232)$ & 0.050 \\
\hline Double-vessel CAD & $28.3(129 / 456)$ & $25.4(57 / 224)$ & $31.0(72 / 232)$ & 0.19 \\
\hline Triple-vessel CAD & $7.9(36 / 456)$ & $6.3(14 / 224)$ & $9.5(22 / 232)$ & 0.20 \\
\hline LM CAD & $3.7(17 / 456)$ & $4.0(9 / 224)$ & $3.4(8 / 232)$ & 0.75 \\
\hline CTO in non-target lesion & $12.9(59 / 456)$ & $13.4(30 / 224)$ & $12.5(29 / 232)$ & 0.78 \\
\hline Planned PCI after discharge, $\%(n / N)$ & $13.4(61 / 456)$ & $12.9(29 / 224)$ & $13.8(32 / 232)$ & 0.79 \\
\hline \multicolumn{5}{|l|}{ Antithrombotic therapy on discharge, \% $(n / N)$ : } \\
\hline Acetylsalicylic acid & $99.3(453 / 456)$ & $99.1(222 / 224)$ & $99.6(231 / 232)$ & 0.54 \\
\hline Clopidogrel & $82.7(79 / 456)$ & $91.5(205 / 224)$ & $74.1(172 / 232)$ & $<0.0001$ \\
\hline Ticagrelor & $10.1(46 / 456)$ & $1.3(3 / 224)$ & $18.5(43 / 232)$ & $<0.0001$ \\
\hline Prasugrel & $7.0(32 / 456)$ & $6.7(15 / 224)$ & $7.3(17 / 232)$ & 0.79 \\
\hline Oral anticoagulant & $6.6(30 / 456)$ & $8.0(18 / 224)$ & $5.2(12 / 232)$ & 0.22 \\
\hline Novel oral anticoagulant & $0.9(4 / 456)$ & $0.4(1 / 224)$ & $1.3(3 / 232)$ & 0.33 \\
\hline
\end{tabular}

${ }^{a}$ On admission. CABG - coronary artery bypass grafting, CTO - chronic total occlusion, eGFR - estimated glomerular filtration rate, LVEF - left ventricular ejection fraction, MI - myocardial infarction, LM CAD - left main coronary artery disease, PCl - percutaneous coronary intervention, Q1-Q3 - quartiles 1 and 3, SA - stable angina, SD - standard deviation, STEMI - ST-segment elevation myocardial infarction, WBC - white blood cells. 
Table II. Procedural characteristics of study population

\begin{tabular}{|c|c|c|c|c|}
\hline Factor & $\begin{array}{l}\text { Study population } \\
\quad(N=456)\end{array}$ & $\begin{array}{l}\text { QCA group } \\
(N=224)\end{array}$ & $\begin{array}{c}\text { Non-QCA group } \\
(N=232)\end{array}$ & $P$-value \\
\hline Total number of procedures, $n$ : & 467 & 228 & 239 & \\
\hline $\mathrm{PCl}$ ad hoc, \% $(n / N)$ & $89.9(420 / 467)$ & $91.7(209 / 228)$ & $88.3(211 / 239)$ & 0.22 \\
\hline Femoral access, \% $(n / N)$ & $69.2(323 / 467)$ & $81.1(185 / 228)$ & $57.7(138 / 239)$ & $<0.0001$ \\
\hline Radial access, \% $(n / N)$ & $30.4(142 / 467)$ & $18.4(42 / 228)$ & $41.8(100 / 239)$ & $<0.0001$ \\
\hline Other access, \% $(n / N)$ & $0.4(2 / 467)$ & $0.4(1 / 228)$ & $0.4(1 / 239)$ & 0.97 \\
\hline Total number of target lesions, $n$ : & 563 & 249 & 314 & \\
\hline Lesions per patient, number, mean \pm SD $(n / N)$ & $1.23 \pm 0.51(563 / 456)$ & $1.13 \pm 0.49(249 / 224)$ & $1.34 \pm 0.54(314 / 232)$ & $<0.0001$ \\
\hline Percent diameter stenosis, mean \pm SD $(n / N)[\%]$ & $83.5 \pm 12.7(563 / 563)$ & $83.6 \pm 12.6(249 / 249)$ & $83.5 \pm 12.7(314 / 314)$ & 0.78 \\
\hline Left main, \% $(n / N)$ & $0.4(2 / 563)$ & $0.4(1 / 249)$ & $0.3(1 / 314)$ & 0.87 \\
\hline Left anterior descending, \% $(n / N)$ & $53.6(302 / 563)$ & $57(142 / 249)$ & $51(160 / 314)$ & 0.15 \\
\hline Left circumflex, \% $(n / N)$ & $25.4(143 / 563)$ & $23.7(59 / 249)$ & $26.8(84 / 314)$ & 0.41 \\
\hline Right coronary artery, \% $(n / N)$ & $20.4(115 / 563)$ & $18.5(46 / 249)$ & $22.0(69 / 314)$ & 0.31 \\
\hline Bypass, \% $(n / N)$ & $0.2(1 / 563)$ & $0.4(1 / 249)$ & $0.0(0 / 314)$ & 0.26 \\
\hline Aorto-ostial, \% $(n / N)$ & $7.3(41 / 563)$ & $8.0(20 / 249)$ & $6.7(21 / 314)$ & 0.54 \\
\hline Bifurcation, \% $(n / N)$ & $16.2(91 / 563)$ & $14.5(36 / 249)$ & $17.5(55 / 314)$ & 0.33 \\
\hline Thrombus, \% $(n / N)$ & $8.7(49 / 563)$ & $10.4(26 / 249)$ & $7.3(23 / 314)$ & 0.19 \\
\hline Thrombectomy, \% $(n / N)$ & $5.2(29 / 563)$ & $7.2(18 / 249)$ & $3.5(11 / 314)$ & 0.047 \\
\hline Restenotic, \% $(n / N)$ & $4.1(23 / 563)$ & $4.0(10 / 249)$ & $4.1(13 / 314)$ & 0.94 \\
\hline Pre-dilatation, \% $(n / N)$ & $95.7(539 / 563)$ & $94.8(236 / 249)$ & $96.5(303 / 314)$ & 0.32 \\
\hline Post-dilatation, \% $(n / N)$ & $56.8(320 / 563)$ & $39.8(99 / 249)$ & $70.4(221 / 314)$ & $<0.0001$ \\
\hline Pre-intervention TIMI flow 0-1, \% $(n / N)$ & $18.1(102 / 563)$ & $17.7(44 / 249)$ & $18.5(58 / 314)$ & 0.81 \\
\hline Post-intervention TIMI flow 3, \% $(n / N)$ & $99.3(559 / 563)$ & $99.6(248 / 249)$ & $99.0(311 / 314)$ & 0.44 \\
\hline Total number of devices, $n$ : & 588 & 253 & 335 & \\
\hline Devices per patient, number, mean \pm SD $(n / N)$ & $1.29 \pm 0.56(588 / 456)$ & $1.14 \pm 0.49(253 / 224)$ & $1.43 \pm 0.61(335 / 232)$ & $<0.0001$ \\
\hline Total device length, mean $\pm \mathrm{SD}(n / N)[\mathrm{mm}]:$ & $20.9 \pm 5.8(588 / 456)$ & $20.3 \pm 5.8(253 / 224)$ & $21.3 \pm 5.8(335 / 232)$ & 0.027 \\
\hline $8 \mathrm{~mm}, \%(n / N)$ & $0.7(4 / 588)$ & $0.8(2 / 253)$ & $0.6(2 / 335)$ & 0.78 \\
\hline $12 \mathrm{~mm}, \%(n / N)$ & $16.5(97 / 588)$ & $17.6(46 / 253)$ & $15.2(51 / 335)$ & 0.34 \\
\hline $18 \mathrm{~mm}, \%(n / N)$ & $36.4(214 / 588)$ & $46.6(118 / 253)$ & $28.7(96 / 335)$ & $<0.0001$ \\
\hline $23 \mathrm{~mm}, \%(n / N)$ & $14.3(84 / 588)$ & $5.5(14 / 253)$ & $20.9(70 / 335)$ & $<0.0001$ \\
\hline $28 \mathrm{~mm}, \%(n / N)$ & $32.1(189 / 588)$ & $30.8(78 / 253)$ & $33.1(111 / 335)$ & 0.55 \\
\hline Nominal device diameter, mean \pm SD $(n / N)[\mathrm{mm}]$ : & $3.0 \pm 0.4(588 / 456)$ & $3.0 \pm 0.4(253 / 224)$ & $3.0 \pm 0.4(335 / 232)$ & 0.25 \\
\hline $2.5 \mathrm{~mm}, \%(n / N)$ & $29.3(172 / 588)$ & $26.9(68 / 253)$ & $31.0(104 / 335)$ & 0.27 \\
\hline $3.0 \mathrm{~mm}, \%(n / N)$ & $36.7(216 / 588)$ & $37.2(94 / 253)$ & $36.4(122 / 335)$ & 0.85 \\
\hline $3.5 \mathrm{~mm}, \%(n / N)$ & $34.0(200 / 588)$ & $36(91 / 253)$ & $32.5(109 / 335)$ & 0.38 \\
\hline Deployment pressure & $13.8 \pm 2.4(588 / 456)$ & $13.8 \pm 2.5(64 / 224)$ & $13.8 \pm 2.4(49 / 232)$ & 0.93 \\
\hline \multicolumn{5}{|l|}{ Procedure characteristics (per procedure): } \\
\hline Glycoprotein IIb/IIla inhibitors, \% $(n / N)$ & $8.6(40 / 467)$ & $7.9(18 / 228)$ & $9.2(22 / 239)$ & 0.61 \\
\hline Cardiogenic shock, \% $(n / N)$ & $0.6(3 / 467)$ & $0.4(1 / 228)$ & $0.8(2 / 239)$ & 0.59 \\
\hline Life-threatening ventricular arrhythmias, \% $(n / N)$ & $0.9(4 / 467)$ & $0.9(2 / 228)$ & $0.8(2 / 239)$ & 0.96 \\
\hline Perforation, \% $(n / N)$ & $0.2(1 / 467)$ & $0.0(0 / 228)$ & $0.4(1 / 239)$ & 0.33 \\
\hline Side branch occlusion, \% $(n / N)$ & $0.9(4 / 467)$ & $0.9(2 / 228)$ & $0.8(2 / 239)$ & 0.96 \\
\hline No-reflow, \% $(n / N)$ & $0.6(3 / 467)$ & $0.4(1 / 228)$ & $0.8(2 / 239)$ & 0.59 \\
\hline \multicolumn{5}{|l|}{ Patient characteristics during hospitalization (per patient): } \\
\hline Additional BMS/DES, \% $(n / N)$ : & $9.0(41 / 456)$ & $5.8(13 / 224)$ & $12.1(28 / 232)$ & 0.019 \\
\hline Index vessel & $6.4(29 / 456)$ & $4.9(11 / 224)$ & $7.8(18 / 232)$ & 0.21 \\
\hline Index lesion & $1.8(8 / 456)$ & $1.7(4 / 224)$ & $1.7(4 / 232)$ & 0.96 \\
\hline
\end{tabular}

\footnotetext{
$\mathrm{PCl}$ - percutaneous coronary intervention.
} 
ble $\mathrm{V})$. The 24-month overall incidence of scaffold thrombosis was 12 (2.6\%) study participants. Acute (0 to $24 \mathrm{~h}$ ), subacute ( $>24 \mathrm{~h}$ to 30 days) and late ( $>30$ days to 1 year) scaffold thrombosis were found in 2 (0.4\%), 8 (1.8\%) and $2(0.4 \%)$ patients respectively. Very late thrombosis was not observed. Independent predictors of occurrence primary endpoint DoCE in 24-month observation were lack of procedural success, lower ejection fraction, anemia, coexisting total chronic occlusions in non-culprit vessel and peripheral artery disease (Figure 1).

\section{Discussion}

Early and long-term clinical outcomes are reported for this all-comers single-center registry of the Absorb BVS. This registry shows the "real-world" clinical practice of BVS implantation in our institution. A strength of this registry is that it includes a significant proportion of high risk patients such as ACS patients (62.3\%), and patients with complex coronary lesions (78.7\%). Additionally, our registry has QCA data for our first 224 treated patients recorded by an independent Core Lab. Moreover, all adverse events were confirmed using source data. Follow-ups were performed based on the National Health Fund records, which provided complete hospitalization information and detailed procedural descriptions for the analyzed period.

Our primary endpoint DoCE was $6.7 \%$ at 12 months and $12.2 \%$ at 24 months. In the European registry, GHOST-EU, the rate of DoCE at 1 year was $10.1 \%$, with a smaller proportion of patients in the European registry having acute coronary syndromes (47.3\%) and a smaller proportion of patients with B2/C lesions (51.2\%) [11]. The German-Austrian Absorb registry (GABI-R) showed a very low rate of DoCE (2.4\%) but had only a short period of observation (6 months), a smaller proportion of patients
Table III. Quantitative coronary angiography analysis

\begin{tabular}{|c|c|}
\hline Factor & $\begin{array}{l}\text { QCA group } \\
(N=224)\end{array}$ \\
\hline \multicolumn{2}{|l|}{ Baseline: } \\
\hline Lesion type A, \% $(n / N)$ & $3.1(7 / 224)$ \\
\hline Lesion type B1, \% $(n / N)$ & $18.2(41 / 224)$ \\
\hline Lesion type B2, \% $(n / N)$ & $55.6(125 / 224)$ \\
\hline Lesion type C, \% $(n / N)$ & $23.1(52 / 224)$ \\
\hline Tortuosity, \% $(n / N)$ & $9.7(22 / 224)$ \\
\hline Bifurcation, \% $(n / N)$ & $14.7(33 / 224)$ \\
\hline Thrombus, \% $(n / N)$ & $17.6(39 / 224)$ \\
\hline Aorto-ostial, \% $(n / N)$ & $10.0(22 / 224)$ \\
\hline Pre-intervention TIMI flow 0-1, \% $(n / N)$ & $18.6(42 / 224)$ \\
\hline Thrombectomy, \% $(n / N)$ & $7.1(16 / 224)$ \\
\hline Predilatation, \% $(n / N)$ & $92.4(207 / 224)$ \\
\hline $\begin{array}{l}\text { Total lesion length, mean } \pm \mathrm{SD}(n / N) \\
{[\mathrm{mm}]}\end{array}$ & $17.1 \pm 9.3(224 / 224)$ \\
\hline $\begin{array}{l}\text { Total device length, mean } \pm \mathrm{SD}(n / N) \\
{[\mathrm{mm}]}\end{array}$ & $20.4 \pm 9.1(224 / 224)$ \\
\hline $\begin{array}{l}\text { MLD before procedure, mean } \pm \text { SD }(n / N) \\
{[\mathrm{mm}]}\end{array}$ & $0.78 \pm 0.54(224 / 224$ \\
\hline $\begin{array}{l}\text { RVD before procedure, mean } \pm \text { SD }(n / N) \\
{[\mathrm{mm}]}\end{array}$ & $2.68 \pm 0.52(224 / 224$ \\
\hline $\begin{array}{l}\text { DS before procedure, mean } \pm \text { SD }(n / N) \\
{[\%]}\end{array}$ & $71.6 \pm 18.4(224 / 224)$ \\
\hline \multicolumn{2}{|l|}{ After procedure: } \\
\hline RVD in scaffold, mean $\pm S D(n / N)[\mathrm{mm}]$ & $2.70 \pm 0.45(224 / 224$ \\
\hline MLD in scaffold, mean $\pm S D(n / N)[\mathrm{mm}]$ & $2.39 \pm 0.47(224 / 224$ \\
\hline Acute gain, mean $\pm \mathrm{SD}(n / N)[\mathrm{mm}]$ & $1.61 \pm 0.61(224 / 224)$ \\
\hline DS in scaffold, mean \pm SD $(n / N)[\%]$ & $11.9 \pm 9.0(224 / 224)$ \\
\hline
\end{tabular}

Table IV. Clinical and procedure characteristics of patients with definite stent thrombosis

\begin{tabular}{lcccccccccc}
\hline Case & $\begin{array}{c}\text { Year of } \\
\text { implan- } \\
\text { tation }\end{array}$ & Age & $\begin{array}{c}\text { Clinical } \\
\text { indication }\end{array}$ & $\begin{array}{c}\text { Predila- } \\
\text { tation }\end{array}$ & $\begin{array}{c}\text { Post-dila- } \\
\text { tation }\end{array}$ & Device size & $\begin{array}{c}\text { Location } \\
\text { of } \text { MI }\end{array}$ & $\begin{array}{c}\text { Time } \\
\text { of ST }\end{array}$ & $\begin{array}{c}\text { Antiplatelet regimen } \\
\text { at the time of ST }\end{array}$ & $\begin{array}{c}\text { Clopidogrel } \\
\text { resistance } \\
\text { (ADP test) }\end{array}$ \\
\hline 1 & 2013 & 66 & STEMI & Yes & No & $2.5 \times 18$ & CX & 87 & ASA + clopidogrel & N/A \\
\hline 2 & 2013 & 59 & NSTE-ACS & Yes & No & $3.0 \times 18 / 3.0 \times 28$ & LAD & 5 & ASA + clopidogrel & Yes \\
\hline 3 & 2013 & 36 & SA & No & No & $3.5 \times 28$ & LAD & 1 & ASA + clopidogrel & No \\
\hline 4 & 2014 & 52 & SA & Yes & Yes & $2.5 \times 18$ & LAD & 3 & ASA + clopidogrel & Yes \\
\hline 5 & 2014 & 61 & NSTE-ACS & Yes & Yes & $3.5 \times 18$ & LAD & 10 & ASA + clopidogrel & N/A \\
\hline 6 & 2014 & 63 & STEMI & Yes & Yes & $3.0 \times 28$ & LAD & 4 & ASA + clopidogrel & Yes \\
\hline 7 & 2014 & 51 & STEMI & Yes & No & $3.0 \times 18$ & LAD & 1 & ASA + clopidogrel & Yes \\
\hline 8 & 2014 & 51 & NSTE-ACS & Yes & Yes & $2.5 \times 28$ & LAD & 62 & ASA + prasugrel & N/A \\
\hline 9 & 2014 & 60 & NSTE-ACS & Yes & No & $2.5 \times 18$ & LCX & 15 & ASA + clopidogrel & No \\
\hline 10 & 2014 & 68 & STEMI & Yes & Yes & $2.5 \times 28$ & LAD & 11 & ASA + clopidogrel & Yes \\
\hline 11 & 2015 & 65 & SA & Yes & Yes & $2.5 \times 28$ & RCA & 10 & ASA + prasugrel & N/A \\
\hline 12 & 2015 & 60 & STEMI & Yes & Yes & $2.5 \times 23 / 3.0 \times$ & LAD & 15 & ASA + clopidogrel & Yes
\end{tabular}

ASA - acetylsalicylic acid, LCX - left circumflex, LAD - left anterior descending artery, MI-myocardial infarction, N/A - not available, NSTE-ACS - non-ST-segment elevation acute coronary syndrome, RCA - right coronary artery, SA - stable angina, ST - scaffold thrombosis, STEMI - ST-segment elevation myocardial infarction. 
Table V. Long-term outcomes of study population

\begin{tabular}{|c|c|c|c|c|}
\hline Factor & $\begin{array}{l}\text { Study population } \\
\qquad(N=456)\end{array}$ & $\begin{array}{l}\text { QCA group } \\
(N=224)\end{array}$ & $\begin{array}{l}\text { Non-QCA group } \\
\quad(N=232)\end{array}$ & $P$-value \\
\hline \multicolumn{5}{|l|}{ Primary endpoints: } \\
\hline \multicolumn{5}{|l|}{ DoCE: } \\
\hline 12-month, \% $(n / N)$ & $6.7(26 / 387)$ & $6.7(15 / 224)$ & $6.7(11 / 163)$ & 0.98 \\
\hline 24-month, \% $(n / N)$ & $12.2(31 / 254)$ & $11.6(23 / 199)$ & $14.5(8 / 55)$ & 0.55 \\
\hline \multicolumn{5}{|l|}{ Secondary endpoints: } \\
\hline \multicolumn{5}{|l|}{ PoCE: } \\
\hline 12-month, \% $(n / N)$ & $12.4(48 / 387)$ & $11.2(25 / 224)$ & $14.1(23 / 163)$ & 0.38 \\
\hline 24-month, \% $(n / N)$ & $20.1(51 / 254)$ & $19.1(38 / 199)$ & $23.6(13 / 55)$ & 0.46 \\
\hline Device success (lesion basis) & $98.7(556 / 563)$ & $98.4(245 / 249)$ & $99.0(311 / 314)$ & 0.49 \\
\hline Procedural success (patient basis) & $96.9(442 / 456)$ & $96.4(216 / 224)$ & $97.4(226 / 232)$ & 0.54 \\
\hline \multicolumn{5}{|l|}{ Clinical endpoints: } \\
\hline \multicolumn{5}{|l|}{ All-cause death: } \\
\hline 12-month, \% $(n / N)$ & $2.3(9 / 387)$ & $2.2(5 / 224)$ & $2.5(4 / 163)$ & 0.89 \\
\hline 24-month, \% $(n / N)$ & $5.5(14 / 254)$ & $5.0(10 / 199)$ & $7.3(4 / 55)$ & 0.52 \\
\hline \multicolumn{5}{|l|}{ Cardiac death: } \\
\hline 12-month, \% (n/N) & $2.1(8 / 387)$ & $2.5(4 / 163)$ & $1.8(4 / 224)$ & 0.65 \\
\hline 24-month, \% $(n / N)$ & $4.7(12 / 254)$ & $4.0(8 / 199)$ & $7.3(4 / 55)$ & 0.31 \\
\hline \multicolumn{5}{|l|}{ All MI: } \\
\hline 12-month, \% (n/N) & $6.5(25 / 387)$ & $4.9(11 / 224)$ & $8.6(14 / 163)$ & 0.15 \\
\hline 24-month, \% (n/N) & $6.7(17 / 254)$ & $5.5(11 / 199)$ & $10.9(6 / 55)$ & 0.16 \\
\hline \multicolumn{5}{|l|}{ TV-MI: } \\
\hline 12-month, \% $(n / N)$ & $4.4(17 / 387)$ & $4.9(11 / 224)$ & $3.7(6 / 163)$ & 0.56 \\
\hline 24-month, \% $(n / N)$ & $5.5(14 / 254)$ & $5.5(11 / 199)$ & $5.5(3 / 55)$ & 0.98 \\
\hline \multicolumn{5}{|l|}{ ID-TLR: } \\
\hline 12-month, \% $(n / N)$ & $4.7(18 / 387)$ & $5.4(12 / 224)$ & $3.7(6 / 163)$ & 0.44 \\
\hline 24-month, \% (n/N) & $7.9(20 / 254)$ & $8.0(16 / 199)$ & $7.3(4 / 55)$ & 0.85 \\
\hline \multicolumn{5}{|l|}{ ID-TVR: } \\
\hline 12-month, \% (n/N) & $5.7(22 / 387)$ & $6.3(14 / 224)$ & $4.9(8 / 163)$ & 0.57 \\
\hline 24-month, \% (n/N) & $8.7(22 / 254)$ & $9.0(18 / 199)$ & $7.3(4 / 55)$ & 0.68 \\
\hline \multicolumn{5}{|l|}{ Definite scaffold thrombosis: } \\
\hline Acute, $\%(n / N)$ & $0.4(2 / 456)$ & $0.9(2 / 224)$ & $0.0(0 / 232)$ & 0.15 \\
\hline Subacute, \% $(n / N)$ & $1.8(8 / 456)$ & $2.2(5 / 224)$ & $1.3(3 / 232)$ & 0.45 \\
\hline Late, $\%(n / N)$ & $0.4(2 / 456)$ & $0.4(1 / 224)$ & $0.4(1 / 232)$ & 0.98 \\
\hline Very late, \% $(n / N)$ & $0.0(0 / 254)$ & $0.0(0 / 199)$ & $0.0(0 / 55)$ & 0.99 \\
\hline
\end{tabular}

DoCE - device-oriented composite endpoint, ID-TLR - ischemia-driven target lesion revascularization, ID-TVR - ischemia-driven target vessel revascularization, MI-myocardial infarction, POCE - patient-oriented composite endpoint, SA - stable angina, TV-MI - target vessel myocardial infarction.

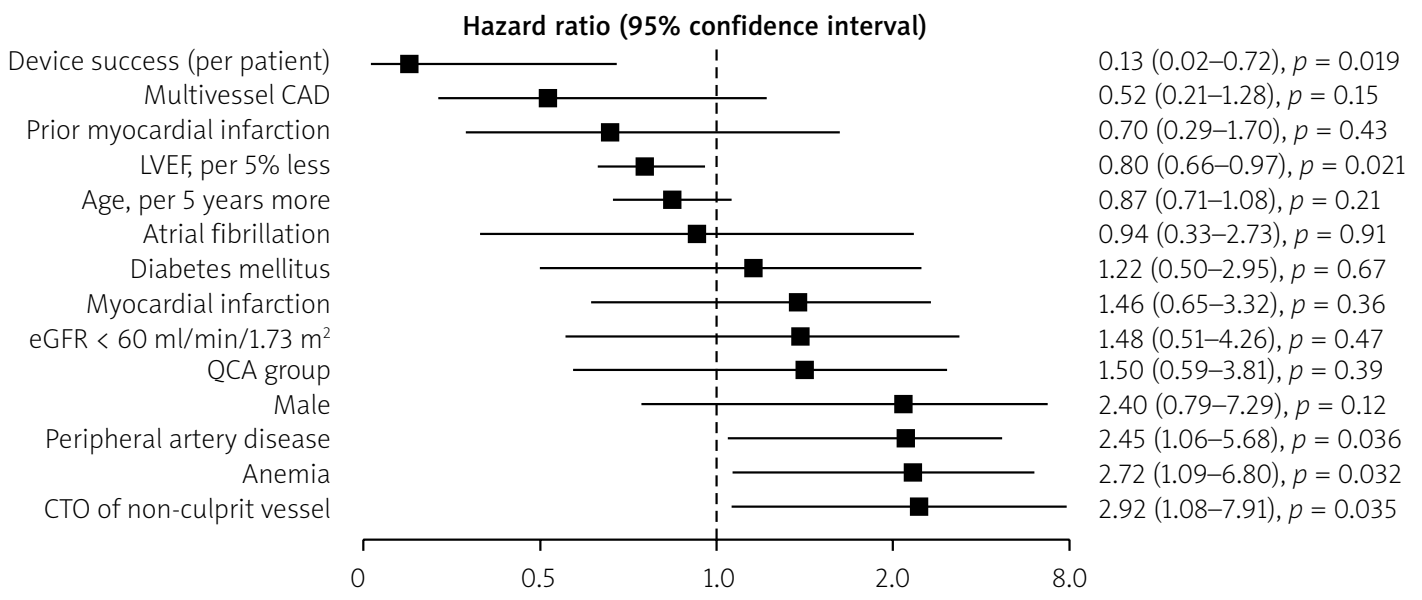

Figure 1. Forest plot of independent predictors of 24-month target lesion failure in study population

CAD - coronary artery disease, CTO - chronic total occlusion, eGFR - estimated glomerular filtration rate, LVEF - left ventricular ejection fraction, QCA - quantitative coronary analysis. 
with ACS (51.5\%) and a smaller proportion of patients with complex coronary lesions (36.5\%) [12]. A meta-analysis of 5 randomized studies (ABSORB II, ABSORB Japan, ABSORB China, TROFI II, EVERBIO) reported a DoCE value of $8.3 \%$ after 2 years. As opposed to registries, trial patients were enrolled according to selection criteria. There was a smaller proportion of patients with acute coronary syndromes (36.6\%) and a smaller proportion with B2/C level changes (58.3\%) [13]. Overall, the clinical outcomes in our registry demonstrate acceptable rates of target lesion failure at 12- and 24-month follow up, especially taking the high number of patients with ACS and with complex lesions into consideration. These good results in a relatively high-risk population at our center may be attributable to the extensive experience of the operators, who perform more than 250 angioplasties per year.

At present, one of the most significant disadvantages of implanting BVS instead of metal stents is the greater risk of in-scaffold thromboses. This includes both early ( 0 to 30 days), late (> 30 days to 1 year) and very late (1 year after) thromboses. In open-access publications the percentage of patients who have developed thrombosis after BVS implantation ranges from $0.0 \%$ to $3.3 \%$ at 6-month follow-up $[14,15]$. According to Collet et al., the main reasons for in-scaffold thrombosis are mechanical damage of the structure of struts occurring during implantation, edge dissections, malapposition of scaffolds and an insufficiently long period of dual antiplatelet therapy (DAPT) [13]. Therefore, none of the main reasons is inherent to the structure of the BVS itself, instead most likely occurring as a result of the management of BVS implantation. Based on the Maintz registry, authors have demonstrated that compliance with a protocol of mandatory pre-dilatation and post-dilatation with high pressure non-compliant balloons during BVS implantations, as well as the selection of vessel-sized BVS, decreases the annual percentage of in-scaffold thrombosis occurrence from $3.3 \%$ to $1 \%(p=0.02)$ [16]. Such an implantation strategy is frequently referred to as Prepare the vessel, Size appropriately and Post-dilate, or PSP.

$2.6 \%$ of patients from our registry developed thrombosis in the 2-year follow-up following BVS implantation. Nine of those patients had a BVS implanted during myocardial infarction, 5 appeared to be lacking post-dilatation and 6 experienced clopidogrel resistance (Table IV). Among study patients, post-dilatation was performed in less than $57 \%$ of cases. One explanation for this fact may be the change in the recommendations for the optimal BVS implantation technique and less rigorous approach to the post-dilatation performance at the beginning of our study. On the other hand, operators could avoid post-dilatation in patients with ACS to prevent the no-reflow phenomenon. Moreover, in patients with scaffold thrombosis, BVSs with a diameter of $2.5 \mathrm{~mm}$ were used in 7 cases. Data from our study confirm that PSP is a crucial factor for preventing thrombosis in BVS.

Considering that the majority of patients in the registry had complex lesions (78.7\% had B2/C level lesions) and the period of observation of 24 months, our results are favorable in comparison to those from other BVS registries. However, the BVS does not compare favorably to second generation DES especially when it comes to thrombosis incidence. A larger cross-section of the scaffolding $(150 \mu \mathrm{m})$ in comparison to commonly used DES $(65-91 \mu \mathrm{m})$ may be one of the contributors to this observation, particularly in small vessels. Thicker struts require a longer period of time to cover themselves with neointima. Additionally, they may disturb endothelial shear which contributes to thrombosis incidence [17]. Late thrombosis may also develop during late discontinuity if parts of the struts remaining uncovered with endothelium come into contact with blood [18]. Peri-strut low-intensity areas visible in OCT may cause hypersensitivity reactions and inflammatory responses during polymer degradation [19]. It is unknown whether such observed late changes are typical for the Absorb BVS or for the whole class of BVS composed of poly-L-lactide acid (PLLA) products. It is estimated that bioresorption of BVS continues for more than 3 years, which is greater than the 1 year currently recommended for double antiplatelet therapy [19]. The ABSORB III trial and COLLET meta-analysis have shown that discontinuation of DAPT after 12 months could be a reason for late in-scaffold thrombosis [13]. In the ABSORB II study, late and very late thromboses were not observed in 63 patients who continued DAPT until the third year of observation [8]. Amongst the 12 patients in our registry who developed thrombosis, around $50 \%$ had proven clopidogrel resistance. In our registry population 12-month long DAPT was recommended. All patients with thrombosis diagnosed were treated with ticagrelor or prasugrel, resulting in no thrombosis re-assigned. Initially in our registry only $17 \%$ of patients received ticagrelor and prasugrel as DAPT. In the light of current knowledge, the debate on prolonging the time of DAPT after BVS implantation seems to be crucial, especially given that despite the withdrawal of the product from sale, there remains a significant number of patients who live with an implanted Absorb BVS.

There were significant differences in baseline clinical, procedural characteristics and pharmacotherapy between the first and second BVS implantation period. As proven in large-scale registries, this may be the result of initially performing the procedure in less complex cases during the process of adoption of the new technology [20]. Despite this, the group treated in the first period consisted of relatively complex patients as $78.7 \%$ of them suffered type B2 or C class lesions (according to the ACC/AHA classification). Despite the small percentage of 
post-dilatations performed in the first period (39.8\%) the reference vessel diameter (RVD) was $2.70 \pm 0.45 \mathrm{~mm}$, with acute gain of $1.61 \pm 0.61 \mathrm{~mm}$. Nevertheless, the incidence of adverse cardiovascular events was similar in both periods.

The major limitation of this study is that it is a single arm, open label single center registry without comparison to drug-eluting stents. Results may differ between the QCA group and non-QCA group due to the fact that assignment to these groups was not random. The first 224 patients treated with BVS were subjected to QCA. Quantitative angiographic assessment of the entire group would improve the value of the analysis. Another limitation is the lack of a standardized operating protocol for BVS implantation in the registry. Operators performed their implantations according to the recommendations of the company at that time. Additionally, we could not verify whether all the patients had been on DAPT for one year, as recommended, as there were no data in the registry on DAPT cessation or continuation.

\section{Conclusions}

The Absorb BVS was successfully and safely implanted in an unselected group of patients. Scaffold thrombosis developed predominantly in patients treated due to acute coronary syndromes. Thus, in this hypothesis-generating ZABRZE-BVS registry, we provide evidence for the safety and efficacy of the BVS in an all-comers population.

\section{Conflict of interest}

The authors declare no conflict of interest.

\section{References}

1. Hawranek M, Desperak P, Ciślak A, et al. Safety and efficacy of a second-generation coronary sirolimus-eluting stent with biodegradable polymers in daily clinical practice: a 12-month follow-up of the ALEX registry. Coron Artery Dis 2016; 27: 89-94.

2. Palmerini T, Biondi-Zoccai G, Della Riva, D, et al. Stent thrombosis with drug-eluting and bare-metal stents: evidence from a comprehensive network meta-analysis. Lancet 2012; 379: 1393-402.

3. Lange RA, Hillis LD. Second-generation drug-eluting coronary stents. N Engl J Med 2010; 362: 1728-30.

4. Alfonso F, Fernandez C. Second-generation drug-eluting stents. Moving the field forward. J Am Coll Cardiol 2011; 58: 26-9.

5. Serruys PW, Onuma Y, Dudek D, et al. Evaluation of the second generation of a bioresorbable everolimus-eluting vascular scaffold for the treatment of de novo coronary artery stenosis: 12-month clinical and imaging outcomes. J Am Coll Cardiol 2011; 58: 1578-88.

6. Onuma Y, Serruys PW, Gomez J, et al. Comparison of in vivo acute stent recoil between the bioresorbable everolimus-eluting coronary scaffolds (revision 1.0 and 1.1) and the metallic everolimus-eluting stent. Catheter Cardiovasc Interv 2011; 78: 3-12.

7. Dudek D, Onuma Y, Ormiston JA, et al. Four-year clinical follow-up of the ABSORB everolimus-eluting bioresorbable vascu- lar scaffold in patients with de novo coronary artery disease: the ABSORB trial. Eurolntervention 2012; 7: 1060-1.

8. Serruys PW, Chevalier B, Sotomi Y, et al. Comparison of an everolimus-eluting bioresorbable scaffold with an everolimus-eluting metallic stent for the treatment of coronary artery stenosis (ABSORB II): a 3 year, randomised, controlled, single-blind, multicentre clinical trial. Lancet 2016; 388: 2479-91.

9. Windecker S, Kolh P, Alfonso F, et al. 2014 ESC/EACTS Guidelines on myocardial revascularization: The Task Force on Myocardial Revascularization of the European Society of Cardiology (ESC) and the European Association for Cardio-Thoracic Surgery (EACTS) Developed with the special contribution of the European Association of Percutaneous Cardiovascular Interventions (EAPCI). Eur Heart J 2014; 35: 2541-619.

10. Cutlip DE, Windecker S, Mehran R, et al. Clinical end points in coronary stent trials: a case for standardized definitions. Circulation 2007; 115: 2344-51.

11. Capodanno D, Gori T, Nef H, et al. Percutaneous coronary intervention with everolimus-eluting bioresorbable vascular scaffolds in routine clinical practice: early and midterm outcomes from the European multicentre GHOST-EU registry. Eurolntervention 2015; 10: 1144-53.

12. Nef HM, Wiebe J, Kastner J, et al. Everolimus-eluting bioresorbable scaffolds in patients with coronary artery disease: results from the German-Austrian ABSORB RegIstRy (GABI-R). Eurolntervention 2017; 13: 1311-8.

13. Collet C, Asano T, Miyazaki Y, et al. Late thrombotic events after bioresorbable scaffold implantation: a systematic review and meta-analysis of randomized clinical trials. Eur Heart J 2017; 38: 2559-66.

14. Reczuch K, Milewski K, Wąsek W, et al. Bioresorbable scaffolds in the treatment of coronary artery disease. Expert consensus statement of the Association of Cardiovascular Interventions of the Polish Cardiac Society (ACVI PCS). Kardiol Pol 2017; 75: 817-35.

15. Rzeszutko $t$, Siudak Z, Tokarek T, et al. Twelve months clinical outcome after bioresorbable vascular scaffold implantation in patients with stable angina and acute coronary syndrome. Data from the Polish National Registry. Adv Interv Cardiol 2016; 12: 108-15.

16. Puricel $S$, Cuculi F, Weissner $M$, et al. Bioresorbable coronary scaffold thrombosis: multicenter comprehensive analysis of clinical presentation, mechanisms, and predictors. J Am Coll Cardiol 2016; 67: 921-31.

17. Tenekecioglu E, Poon EK, Collet C, et al. The nidus for possible thrombus formation: insight from the microenvironment of bioresorbable vascular scaffold. JACC Cardiovasc Interv 2016; 9 : 2167-8.

18. Sotomi Y, Suwannasom P, Serruys PW, et al. Possible mechanical causes of scaffold thrombosis: insights from case reports with intracoronary imaging. Eurolntervention 2017; 12: 1747-56.

19. Otsuka F, Pacheco E, Perkins LE, et al. Long-term safety of an everolimus-eluting bioresorbable vascular scaffold and the cobalt-chromium XIENCE V stent in a porcine coronary artery model. Circ Cardiovasc Interv 2014; 7: 330-42.

20. Rzeszutko $Ł$, Tokarek T, Siudak Z, et al. Patient profile and periprocedural outcomes of bioresorbable vascular scaffold implantation in comparison with drug-eluting and bare-metal stent implantation. Experience from ORPKI Polish National Registry 2014-2015. Adv Interv Cardiol 2016; 12: 321-8. 